\title{
Practical genetics series introduction: Information overload, a double-edged sword
}

European Journal of Human Genetics (2006) 14, 889. doi:10.1038/sj.ejhg.5201692

Clinicians dealing with genetic issues - be they paediatricians, geneticists and increasingly, general practitioners - now rely heavily on information technology to aid their clinical consultations.

Never before in history has information been so readily available to us, and its exponential rise continues unabated. With multiple media formats, inexorably led by the internet, comes the problem of assimilating this plethora of data, especially as much of it is irrelevant. The resulting modern day phenomenon often referred to as 'technostress', can best be described as a state of being controlled by information technology rather than being empowered by it.

The average clinical geneticist will spend several hours preparing a clinic and typically needs to consult a myriad of sources including the family tree, the patient's test results, and in the case of rare conditions, specialist websites or databases such as OMIM (Online Mendelian Inheritance in Man - www.ncbi.nlm.nih.gov), Orphanet (www.orpha.net), GeneTests (www.geneclinics.org), the Winter-Baraitser Dysmorphology or Possum Databases. Questions then arise as to the availability of genetic testing for a condition, where this may take place and at what cost. Moreover, what are the latest diagnostic criteria and are their evidenced-based management guidelines available?

When I was asked to resurrect the Practical Genetics series I found myself questioning the purpose of these mini articles. Some pieces were excellent in conveying their message while others fell wide of the mark. Faced with reinventing this section we decided to rebrand the term 'Practical' and aim the articles squarely at busy clinicians already burdened with multiple sources of information. We were exacting in our requirements by wishing to create a 'One-Stop-Shop' information resource. Relevant details on a given genetic disorder would be served up concisely with the latest diagnostic criteria and management guidelines displayed in a visually appealing format. The article would objectively debate the background science and its clinical applications (each article then being peer-reviewed by experts in the field) and discuss briefly future developments. Oh, and by the way, each article had to be written by a leading authority in the field!

I believe we have succeeded in each of these aims and I am proud to present the relaunch of the series beginning with Rett syndrome. If you turn to page 896 of this issue you will immediately be faced with an 'In brief' box listing a summary of the key elements of the subject (eg Rett syndrome). Beside this, an algorithm/decision chart to aid diagnosis is typically presented. We believe this general format to be the most appealing, permitting the reader to judge in an instant whether the article will be relevant to their needs.

It is not easy to decide how to prioritise which of the 4000 or so genetic disorders to commission first but, in partnership with Orphanet, we have begun the process in earnest. To mention just a few examples we will cover in forthcoming issues the following; Proteus, Joubert, Rubinstein-Taybi, Sotos, Tuberous Sclerosis, Neurofibromatosis, Börjeson-Forssman-Lehman and the otopalatodigital syndrome spectrum disorders.

The partnership with Orphanet is a unique one in that each Practical Genetics article will be subject to open access and will thus be free to download; a prerequisite if the series is to succeed. Each paper will also be archived on the Orphanet website (www.orpha.net) from which they can be easily searched out and where they will be interfaced with other relevant information for the clinician such as a list of clinical laboratories offering a test, a list of specialised clinics, patient organisations, on-going clinical trials, patient registries and research projects.

Finally, I hope that our readers, clinical or otherwise will find the Practical Genetics series useful. Furthermore, we will be grateful for comments, good or bad so that we may continuously improve the content and thereby alleviate you, at least in part, of your technostress.

Philip L Beales 